\title{
ARTICLE OPEN Room-Temperature Graphene-Nanoribbon Tunneling Field-Effect Transistors
}

\author{
Wan Sik Hwang ${ }^{1,2,3 *}$, Pei Zhao ${ }^{2}$, Sung Geun Kim ${ }^{4}$, Rusen Yan ${ }^{1}$, Gerhard Klimeck ${ }^{4}$, Alan Seabaugh ${ }^{2}$, Susan K. Fullerton-Shirey ${ }^{2,5}$,
} Huili Grace Xing (iD ${ }^{1,2,6}$ and Debdeep Jena ${ }^{1,2,6 *}$

Controlled, tunable, and reversible negative-differential resistance (NDR) is observed in lithographically defined, atomically thin semiconducting graphene nanoribbon (GNR)-gated Esaki diode transistors at room temperature. Sub-10 nm-wide GNRs patterned by electron-beam lithography exhibit semiconducting energy bandgaps of $\sim 0.2 \mathrm{eV}$ extracted by electrical conductance spectroscopy measurements, indicating an atomically thin realization of the electronic properties of conventional 3D narrowbandgap semiconductors such as InSb. A $p-n$ junction is then formed in the GNR channel by electrostatic doping using graphene side gates, boosted by ions in a solid polymer electrolyte. Transistor characteristics of this gated GNR $p-n$ junction exhibit reproducible and reversible NDR due to interband tunneling of carriers. All essential experimentally observed features are explained by an analytical model and are corroborated by a numerical atomistic simulation. The observation of tunable NDR in GNRs is conclusive proof of the existence of a lithographically defined bandgap and the thinnest possible realization of an Esaki diode. It paves the way for the thinnest scalable manifestation of low-power tunneling field-effect transistors (TFETs).

npj 2D Materials and Applications (2019)3:43; https://doi.org/10.1038/s41699-019-0127-1

\section{INTRODUCTION}

The single-atom thickness of a two-dimensional (2D) sheet of graphene represents the ultimate limit of how thin one can make an electronically active material. ${ }^{1-3}$ At present, there is no known way to scale a three-dimensional (3D) semiconductor to such a thickness. Structurally, this is not possible, because the chemical bonds are inherently 3D and a unit cell of the crystal cannot be completed in 2D. ${ }^{4}$ Electronically, as a conventional 3D-crystal semiconductor is made thinner to approach the single-atom thickness limit, quantum confinement of electron states increases the energy bandgap. ${ }^{4}$ As graphene has a two-atom basis, the onsite energies for an electron for the A- and B-atom sites are identical (both are carbon atoms) and the energy bandgap is zero. The zero gap prevents an appreciable modulation of the electronic conductivity upon sweeping the Fermi level through the Dirac point, preventing the realization of traditional Boolean On/Off electronic transistor switches. Nevertheless, several devices that exploit the uniqueness of the bandstructure and the density of states of graphene have been theoretically envisioned and experimentally demonstrated. ${ }^{5,6}$

How can the bandgap be opened in an atomically thin material? One way is to break the chemical symmetry of graphene. This is achieved, e.g., in hexagonal boron nitride $(\mathrm{hBN})$, where $\mathrm{B}$ and $\mathrm{N}$ are the nearest-neighbor atoms that straddle carbon in the same row of the periodic table. hBN is the compound-semiconductor analog of graphene, where the broken chemical symmetry due to the different on-site energies for the basis atoms opens a bandgap $\sim 5 \mathrm{eV} .{ }^{7,8}$ Because of the large energy gap and chemical inertness, hBN has been used as an insulating barrier for atomically flat and clean van-der-Waals heterointerfaces. ${ }^{9-11}$ It is possible that in the future similar atomically thin compound materials with bandgaps smaller than hBN will be discovered. A number of gapped semiconductors with broken chemical symmetry exist for thicknesses of tri-atomic layers and beyond, such as the threeatom-thick $\mathrm{MoS}_{2}$ family, the four-atom-thick GaSe family, etc. ${ }^{12-14}$ However, as the electrostatic scaling length of a transistor channel goes as $\lambda=\sqrt{\frac{\varepsilon_{\mathrm{s}}}{\varepsilon_{\mathrm{ox}}} t_{\mathrm{s}} t_{\mathrm{ox}}}$ where $\lambda$ is the electrostatic scaling length, $\varepsilon_{\mathrm{s}}$ and $\varepsilon_{\mathrm{ox}}$ are the permittivities of semiconductor and gate oxide, and $t_{\mathrm{s}}$ and $t_{\mathrm{ox}}$ are the thicknesses of the semiconductor channel and the gate oxide, respectively; the thickness of the channel itself will soon become the limiting factor, for which a one-atom-thick material trumps all others.

If chemical symmetry were to be preserved, other symmetries need to be broken to open gaps, e.g., by starting from zero-gap bilayer graphene (no more an atomically thin material) and applying a vertical electric field opens a bandgap. ${ }^{15,16}$ Alternately, a gap can be opened by letting the atomic sites pucker out of plane. For example, this geometric symmetry breaking opens bandgaps in phosphorene. ${ }^{17,18}$ Carbon nanotubes (CNTs) allow a way to break the cylindrical symmetry by requiring the electron wavefunction to satisfy periodic boundary conditions around the circumference. Depending on the chiral wrapping (armchair vs. zig-zag) and the radius, the density of electronic states of carbon nanotubes change from metallic to semiconducting. ${ }^{19}$ Semiconducting nanotubes have been used to demonstrate highly scaled field-effect transistors (FETs) that help fight short-channel degradation because of exceptional electrostatic control in small geometries. ${ }^{20}$ Nanotubes remain highly attractive for electronic switches. Significant progress has been made in the separation of semiconducting and metallic tubes. ${ }^{21}$ Techniques are being developed to assemble them in specified locations. A lack of precise control over the diameter and chirality results in a lack of control over the bandgap, which is required to make identical electronic switches in large numbers. For large-area applications

\footnotetext{
${ }^{1}$ Department of Electrical and Computer Engineering, Cornell University, Ithaca, NY 14853, USA. ${ }^{2}$ Department of Electrical Engineering, University of Notre Dame, Notre Dame, IN 46556, USA. ${ }^{3}$ Department of Materials Engineering, Korea Aerospace University, Goyang 10540, Korea. ${ }^{4}$ Department of Electrical and Computer Engineering, Purdue University, West Lafayette, IN 47906, USA. ${ }^{5}$ Department of Chemical and Petroleum Engineering, University of Pittsburgh, Pittsburgh, PA 15213, USA. ${ }^{6}$ Department of Materials Science and Engineering, Cornell University, Ithaca, NY 14853, USA. *email: whwang@kau.ac.kr; djena@cornell.edu
} 
where high-performance is not necessary, (CNT) FETs are making progress. $^{22}$

Transistor on/off switching characteristics that beat the fundamental room-temperature "Boltzmann" limit of $(\mathrm{kT} / \mathrm{q}) \cdot \ln (10)$ $\sim 60 \mathrm{mV} /$ decade were observed in carbon nanotube channel transistors. ${ }^{23}$ This observation spurred significant recent interest in an energy-efficient tunneling FETs (TFETs) in several 3D material systems. ${ }^{24}$ However, as the transistor size is scaled, the energy bandgaps of 3D tunneling junctions increase due to quantum confinement, ${ }^{4}$ exponentially reducing the on-state tunneling current. This necessitates the use of intrinsically reduced dimensional 2D or one-dimensional (1D) semiconductor materials for scaled TFETs. In an in-plane 2D semiconductor tunnel junction, electrons that are incident on the potential barrier at non- $90^{\circ}$ angles have their tunneling probabilities lowered exponentially; ${ }^{25,26}$ this severe filtering rule reduces the tunneling efficiency, suggesting 1D channels as the most energy-efficient for nanoscale TFETs. This is borne out in benchmarking efforts by the semiconductor industry; a GNR-TFET can outperform all currently conceived low-power switches based on charge, spin, excitons, and negative capacitance under the energy-delay product metric. $^{27}$

Semiconducting graphene nanoribbons (GNRs) with perfectly controlled edges are the most desirable channel materials for TFETs, ${ }^{26-29}$ although not without their own slew of fabrication challenges. Although CNTs are perfectly cylindrical, GNRs lay flat on a substrate and have broken bonds at the edge. This apparent problem may well become a defining feature. It has proven chemically difficult to dope a CNT by the substitution of donor or acceptor atoms, because there are no sites available for covalent bonding. This opportunity is available in GNRs. The possibility of large-area 2D lithographic patterning, the ability to grow largearea graphene, ${ }^{30}$ the excellent heat dissipation on account of laying flat on the substrate, ${ }^{31}$ and all the other reasons described above make GNR TFETs a difficult, yet desirable challenge. In this work, we present the experimental realization of a roomtemperature GNR-TFET. Although the techniques used here, and the performance, are far from what is desired of a highperformance TFET, our demonstration proves conclusively that the dream of a lithographically defined atomically thin 1D TFET platform is not just conceptually sound, it is experimentally feasible. The theoretical analysis of experiments helps identify the research directions that can take this initial demonstration to a truly attractive low-power sub-Boltzmann switch.

\section{RESULTS AND DISCUSSION}

In a recent work, ${ }^{32,33}$ we experimentally showed that lithographically defined atomically thin, $10 \mathrm{~nm}$-wide GNR-FETs (not TFETs) could mimic the electronic behavior of InSb nanowire FETs ${ }^{34}$ in switching, showing the opening of an energy bandgap of the order of $\sim 0.2 \mathrm{eV}$ (the bandgap of InSb is $\sim 0.17 \mathrm{eV}$ ). In Fig. 1a, we show an image of a similarly fabricated sub- $10 \mathrm{~nm}$ back-gated GNR-FET, but now with two additional side gates to enable the Esaki diode formation, and realization of a GNR TFET. The entire graphene region, the long GNR channel, and the side gates are patterned from a monolayer chemical vapor deposition (CVD)grown graphene using electron-beam lithography (Supplementary Fig. S1). The source and drain contacts to the GNR are made to zero-gap wide $2 \mathrm{D}$ graphene regions. ${ }^{32,33}$ Figure $1 \mathrm{~b}$ shows the measured temperature-dependent transfer characteristics and Fig. 1c shows the conductance spectrum measured at $4 \mathrm{~K}$ without side-gate bias for the "control" GNR-FET. The conductance modulation and its temperature dependence indicates the opening of an energy bandgap of $\sim 0.2 \mathrm{eV}$ for the GNR channel. The measured data and its temperature dependence looks remarkably similar to that of an InSb nanowire FET of gap $\sim 0.17 \mathrm{eV}^{34}$ However, as the GNRs are lithographically defined, they remain atomically thin as opposed to carbon nanotubes and InSb nanowires, and can be produced in large and reproducible quantities because of significant advances in lithography. For example, the widths of fins in current Si FinFETs are well below $10 \mathrm{~nm}^{35}$ and such processing tools can be brought to action to mass produce tightly controlled narrow GNRs in the future if there is promise in their behavior.

We then test the effect of side gates to introduce a $p-n$ junction in the GNR channel (Supplementary Fig. S2). Although techniques to chemically dope GNRs have been reported, ${ }^{36-40}$ they have yet to provide the degree of control necessary for the demonstration of the TFET action. We have therefore chosen a solid polymer electrolyte gate in this work to enable the side gates to dope the GNR channel locally (Supplementary Fig. S2). Although both solid polymer electrolytes ${ }^{41}$ and ionic liquids ${ }^{42}$ are frequently used to gate organic and inorganic FETs, we choose a solid polymer electrolyte, because it is solid state and has a low gate to source leakage current. ${ }^{43}$ Furthermore, the electrolytes polyethylene oxide (PEO) and $\mathrm{LiClO}_{4} \mathrm{~S}$ have been used previously to induce charge carrier densities in 2D graphene greater than $n \sim 10^{13}$ $\mathrm{cm}^{-2}{ }^{44}$ Figure $2 \mathrm{a}$ schematically shows how the polarity of the gate sets the channel doping: when a negative voltage is applied to side-gate 2 , the positive Li ions are attracted to the gate pad and the negative $\mathrm{ClO}_{4}$ ions to the GNR channel. This causes the
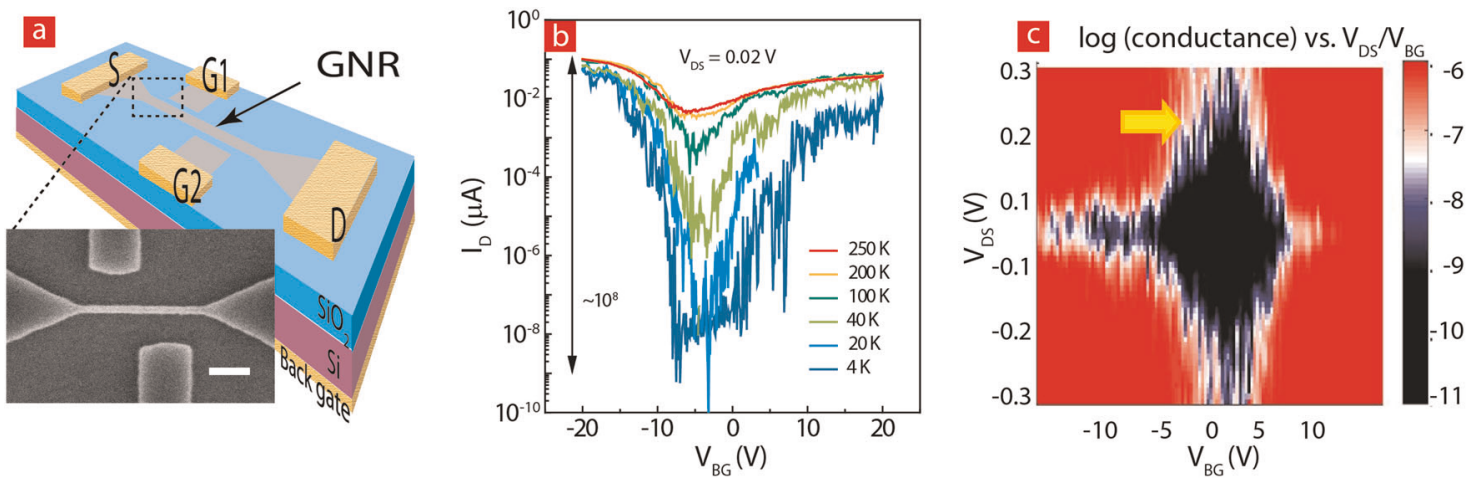

Fig. 1 Graphene nanoribbon (GNR) tunneling field-effect transistors (TFETs). a Schematic of back-gated GNR-TFETs with two side gates that form the $p-n$ junction through electrostatic doping. The insets represent corresponding SEM images of the GNR-TFET. The GNR channel and the graphene side gates are covered by hydrogen silsesquioxane (HSQ). The scale bar is $50 \mathrm{~nm}$. $\mathbf{b}$ Transfer characteristics of a back-gated CVD GNR-FET with GNR of sub-10 $\mathrm{nm}$ width. c Differential conductance map of the GNR as a function of $V_{\mathrm{DS}}$ and $V_{\mathrm{BG}}$ at $4 \mathrm{~K}$ showing an energy bandgap. The arrow shows the gap 

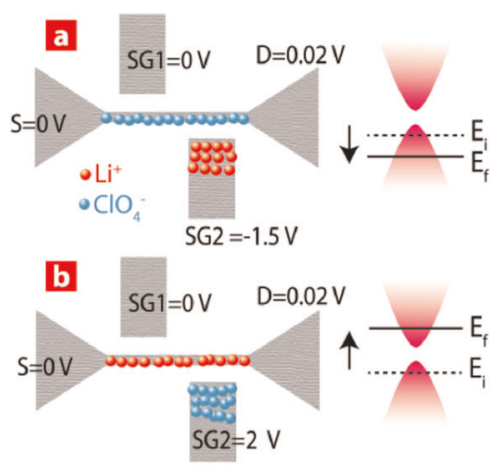
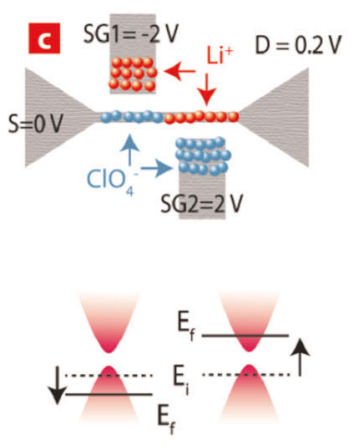

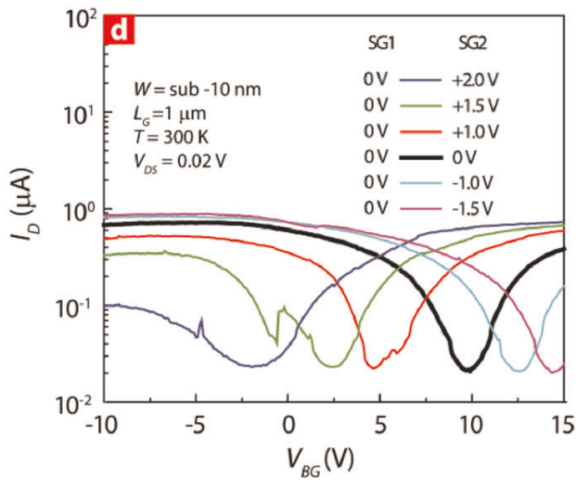

Fig. 2 Electrostatic doping of GNRs using electrolyte gating. Schematic view of electrostatic control of GNRs forming a $p$-type, $\mathbf{b} n$-type, and $\mathbf{c}$, $p-n$ junction in the channel using positive $\left(\mathrm{Li}^{+}\right)$and negative $\left(\mathrm{ClO}_{4}{ }^{-}\right)$ions in polyethylene oxide (PEO). There are equal numbers of positive and negative ions within each conditions $\mathbf{a}-\mathbf{c}$, to satisfy the requirements of electro-neutrality. $\mathbf{d}$ Transfer characteristics of back-gated CVD GNR-FETs demonstrating how the carrier density and carrier type can be modulated using side gates. In contrast to the charge-neutrality point of the GNR-FET in Fig. $1 \mathrm{~b}$ around $-5 \mathrm{~V}$, that of the GNR-FET coated with the solid polymer electrolyte shifted to $10 \mathrm{~V}$, showing that the solid polymer electrolyte dopes the channel even in the absence of a gate voltage
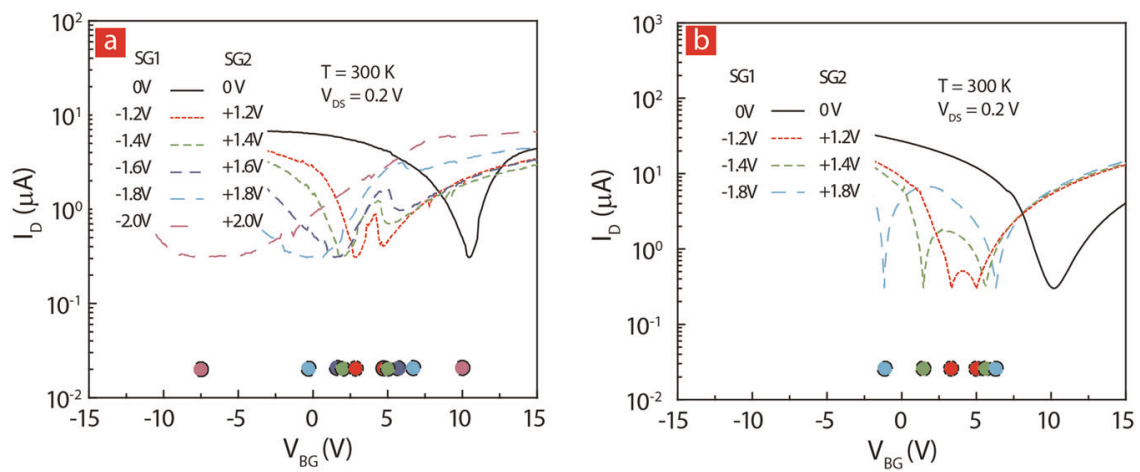

Fig. 3 Formation of $p-n$ junction in the GNRs via electrostatic control using a side gate and a polymer electrolyte. Drain current, $l_{\mathrm{D}}$, vs. backgated, $V_{\mathrm{BG}}$ at different voltages at SG1 and SG2. a Experimental results. $\mathbf{b}$ Analytical modeling results. The dots on the $x$ axes indicate the $V_{\mathrm{BG}}$ where the minima in the transfer characteristics occur. The position of the dots in the $y$ axes are guides to the eye to compare experiments and theory

GNR channel to be doped $p$-type. Then a higher positive back-gate voltage should be necessary to move the Fermi level to its chargeneutrality point. The experimental positive shift in the chargeneutrality point is picked up in the measured room-temperature transfer curve shown in Fig. 2 d. By varying the side-gate voltage, the measured drain current in Fig. $2 d$ shows that the GNR channel can be doped $n$ - or $p$-type by a single side gate. Figure $2 c$ schematically shows that if voltages of opposite polarity are applied to the two side gates, a $p-n$ junction should form in the GNR channel.

Figure 3a shows the transfer characteristics of the device when the side gates are biased with opposite polarity. Two chargeneutrality points appear in the experimentally measured transfer characteristics, proving the formation of a $p-n$ junction in the GNR channel. ${ }^{36}$ Although the measurement was conducted at room temperature, any possibility of Joule heating was ruled out during the operation, based on the low drain voltage of $0.2 \mathrm{~V}$ and excellent heat dissipation of graphene, there might be a very little chance for the heat to affect the device performance. The voltage difference between two current minima increases with the strength of the side-gate bias, as indicated by the circles in the figure. This is simply because the side gates are far more effective in doping the GNR channel than the back gate. The use of ions, their unfettered access to the GNR channel, and their inability to participate in the electron current transport are responsible for this significant enhancement. Figure $3 \mathrm{~b}$ shows the calculated transfer characteristics of the GNR-TFET using a simple analytical model. $^{32}$ The simple model uses thermionic emission and tunneling currents in the channel, appropriately gated with the device capacitances, and captures the entire region of operation of the experimentally measured transfer curve, including the splitting and movement of the charge-neutrality points (see Supplementary Section 3 and Fig. S3). This agreement also indicates the band-like transport in the GNR channel (as opposed to hopping) and the successful realization of a gated GNR $p-n$ junction. The comparison between experiment and model showed that the charge-neutrality points (Fig. 3a) are changing exponentially, while the points (Fig. 3b) are changing linearly. The difference could possibly be attributed to the difference in ion mobility between $\mathrm{Li}^{+}$and $\mathrm{ClO}_{4}{ }^{-}$. Whereas in the modeling work, the $\mathrm{Li}^{+}$and $\mathrm{ClO}_{4}{ }^{-}$ions respond at the same rate to the same potential, in reality the movement is likely to be different because of differences in ion size, mobility, and changes in local dynamics with changing ion concentration. In addition, as shown in this comparison between experiment and model, the on-current density of $6 \mu \mathrm{A} / 10 \mathrm{~nm}$ at a supply voltage of $0.2 \mathrm{~V}$ was experimentally obtained, while that of $40 \mu \mathrm{A} / 10 \mathrm{~nm}$ at a supply voltage of $0.2 \mathrm{~V}$ was extracted by the model. The degraded oncurrent density of the GNR-FET compared with the modeled value was presumably due to the imperfect characteristic of the formed GNR such as edge disorder and defects. This difference of around ten times between experiment and model can also be found in ref. ${ }^{28}$ The currents in Fig. 3 are at a fixed drain bias of $0.2 \mathrm{~V}$; the proof of tunneling is in the $I_{\mathrm{d}}-V_{\mathrm{ds}}$ output characteristics of the GNR TFETs shown in Fig. 4. 

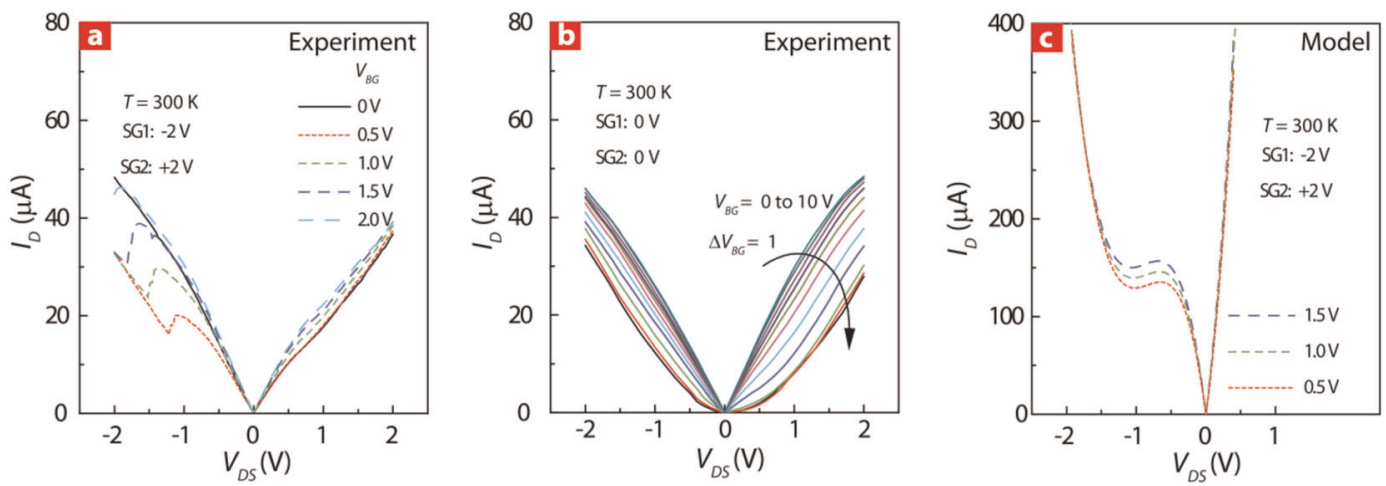

Fig. 4 Output characteristics showing negative-differential resistance (NDR) for electrolyte-gated GNR-TEFTs. Absolute value of the drain current, $I_{\mathrm{D}}$, vs. drain voltage, $V_{\mathrm{DS}}$. a NDR-FET behavior at $V_{\mathrm{DS}}<0$ resulting from the tunneling current through the $p-n$ junction formed by additional side gate and typical FET behavior at $V_{D S}>0$. b Normal FET behavior in $p$-type channel. $\mathbf{c}$ Analytical modeling of NDR-FET behavior

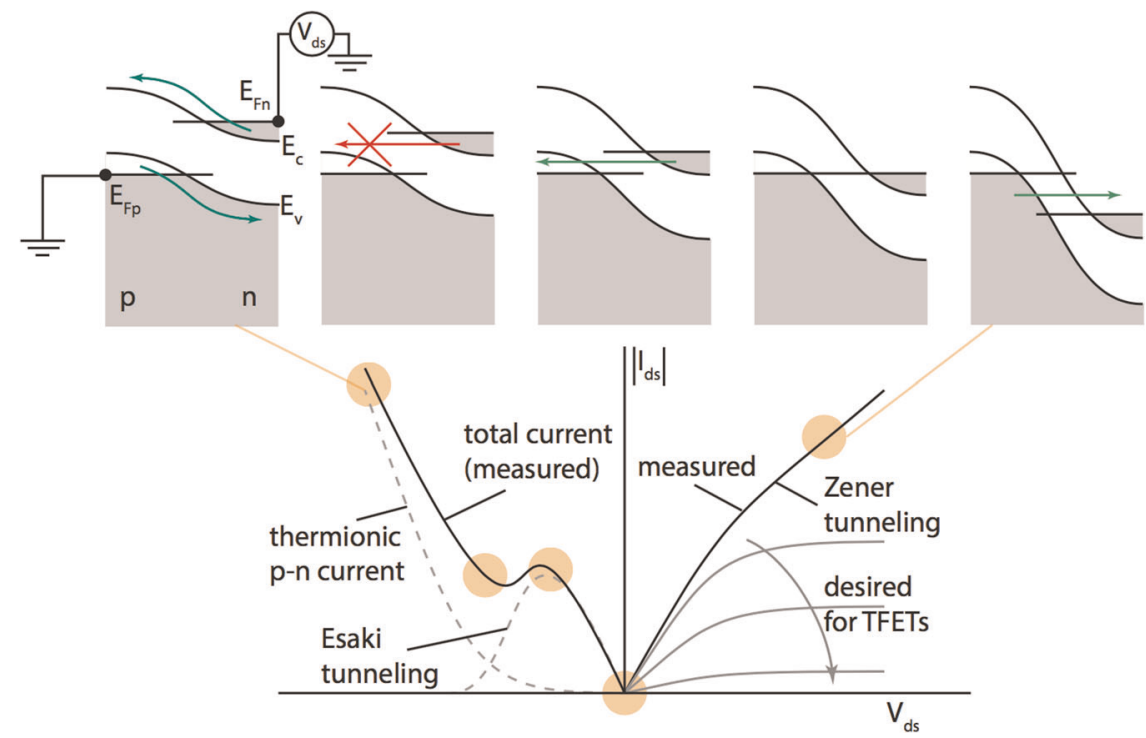

Fig. 5 GNR-TFET operation regimes. The measured experimental data for a fixed back-gate voltage is explained with energy-band diagrams. As side-gate 1 is on the source side and is negatively biased, it makes the GNR source $p$-type, setting the polarity of the GNR $p-n$ junction. When the drain voltage is large and negative, the $p-n$ diode is forward biased and the normal diode thermionic on-current flows. For small forward biases for smaller negative drain voltages, interband Esaki tunneling current flows from the $n$-conduction band states to the $p$ valence-band states, leading to NDR. For positive drain voltages, the GNR is reverse biased and interband Zener tunneling current flows in the device

Figure $4 a$ shows the measured output characteristics of the GNR-TFET measured at room temperature with the two side gates oppositely biased at $\pm 2.0 \mathrm{~V}$, for various back-gate voltage and drain voltages. A clear and reproducible negative-differential resistance (NDR) is observed at room temperature for a range of back-gate voltages and for negative drain bias voltages. The NDR vanishes for positive drain voltages. If the opposite polarity sidegate voltages are removed, the NDR also vanishes for negative drain voltages, as shown in Fig. 4b. The measured roomtemperature characteristics of Fig. $4 \mathrm{~b}$ are that of a $p$-channel GNR-FET with no tunneling, whereas that in Fig. 4a is that of a GNR-TFET. This is the report of NDR in a GNR-TFET. The same analytical model that was used for the transfer characteristics in Fig. $3 b$ also qualitatively reproduces the GNR-TFET output characteristics as shown in Fig. 4c. The fact that the model is able to reproduce all essential features of the device attests to its qualitative accuracy. However, the extreme quantized nature of the GNR electronic states implies that the measured experimental data be compared with an atomistic simulation for quantitative insight.
Before setting up the atomistic model, in Fig. 5 we have explained the measured operation regimes of the GNR-TFET through energy-band diagrams. The NDR due to Esaki tunneling should be observed only for negative drain voltages, because the source-end of the GNR is doped $p$-type by side-gate 1 . Under these conditions, the GNR $p n$ junction is under a small forward bias, the regime for interband Esaki tunneling of electrons from the conduction band in the $n$-side to the empty valence-band states in the $p$-side. The NDR occurs at the drain bias when the band edges go out of alignment. For positive drain voltages, no NDR is expected, because the current is due to interband Zener tunneling, for which there is no chance for band edges to go out of alignment as indicated in Fig. 5. In Fig. 4a, it is also observed that for positive drain voltages the current monotonically increases and does not turn off. The on/off switching in Fig. 4b, although modest, is much more pronounced when the side gates are not biased. This is because to switch the device off, the back gate must stretch the junction tunneling distance and lower the tunneling probability. However, it is unable to so because the far stronger electrostatic doping of the two side gates boosted by the polymer electrolyte overrides this desired back-gate control. Thus, 

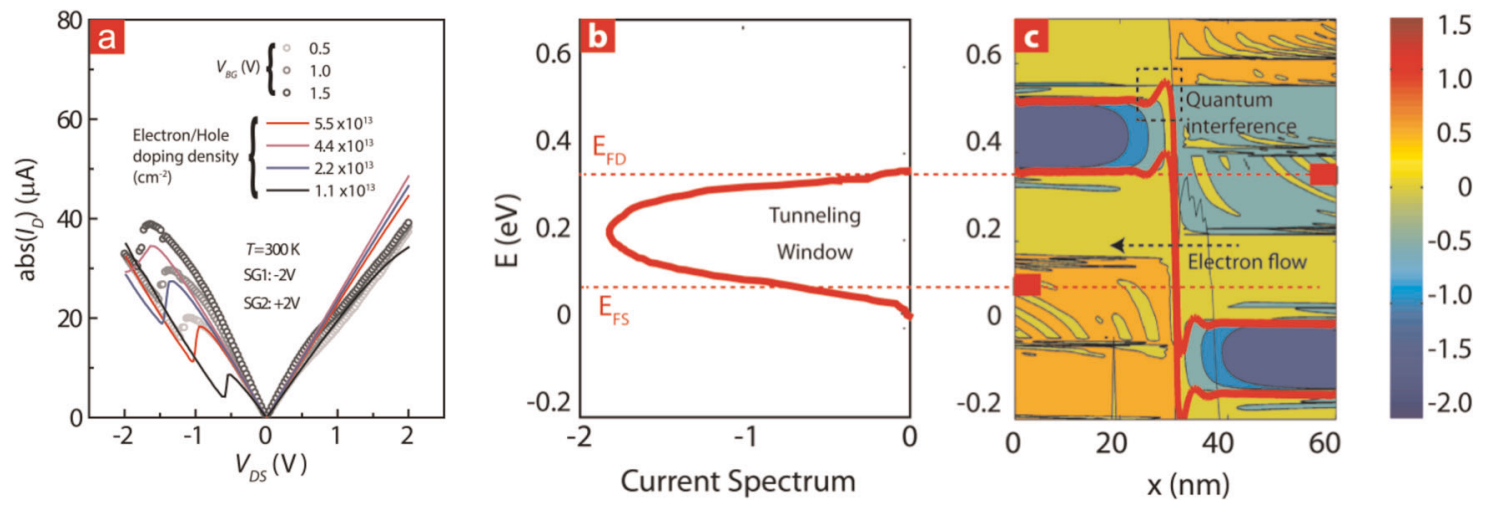

Fig. 6 Numerical atomic simulations of GNR-TEFTs. a Experimentally measured room-temperature GNR-TFET $I_{\mathrm{d}}-V_{\mathrm{ds}}$ characteristics (circles) compared with the numerical atomistic simulation (solid lines). $\mathbf{b}$ The simulated current spectrum of doping density of $1.1 \times 10^{13} / \mathrm{cm}^{2}$ at the NDR peak, which is calculated from the transmission multiplied by $\left(f_{\mathrm{S}}-f_{\mathrm{D}}\right)$, where $f_{\mathrm{S}}$ and $f_{\mathrm{D}}$ are the Fermi functions of the source and the drain contacts. The current mainly flows in the tunneling window between the source and the drain Fermi level. The minus sign of the current spectrum is because the direction of the current is opposite to that of the $x$ axis, whereby the current in a shows only absolute values. c The local density of states shown as a contour plot in a logarithmic scale indicated by the color bar. The conduction and valence-band edges are shown in red solid lines. The source and drain Fermi levels $\left(E_{\mathrm{Fs}} / E_{\mathrm{Fd}}\right)$ are also shown with red dotted lines

although the polymer electrolyte is useful for demonstrating the NDR action, the monotonic increase of the drain current under Zener tunneling also exposes its limitation. To achieve gain in a GNR-TFET, it is desirable that the Zener current saturates and is controlled by the (back) gate voltage as indicated by the gray lines in Fig. 5. These observations point towards the need of direct donor and acceptor doping in the GNR channel without polymer electrolytes in future embodiments, and also underlines the need for narrower GNRs with increased bandgaps in the future. To solidify this qualitative observation, we have employed a comprehensive electrostatic and quantum transport simulation.

The numerical atomistic simulation of the GNR-TFET characteristics is performed using a self-consistent solution of Poisson equation for electrostatics and Schrodinger equation for the electronic bandstructure and quantum transport. For the quantum mechanical portion of the atomistic simulation a $p-d$ tight-binding basis set was used ${ }^{45}$ for determining the energy bandstructure and the transmission formalism was used for calculating the current. Scattering effects are not explicitly included, but lumped into an effective mean-free path and contact resistances (Supplementary Figs S4 and S5). Figure 6a shows the results of the atomistic simulation of the GNR-TFET output characteristics superposed on the measured experimental data. The agreement supports the qualitative analysis and the analytical models, and also offers quantitative insight. For example, the shift in the NDR peak with increasing side-gate doping occurs as the tunneling energy window indicated in Fig. $6 \mathrm{~b}$ is widened. The energyresolved current spectrum indicates the peak current flows near the middle of the tunneling window. Although this feature is similar to the increase in Esaki tunneling current as a pn diode is doped heavier, the energy-resolved local density of states (LDOS) in Fig. $6 \mathrm{c}$ shows a very abrupt tunnel junction with reflections and interference of electron waves occurring over $\sim 4 \mathrm{~nm}$ as visible in the LDOS oscillations. Such features are not captured easily in a typical effective mass Wentzel-Kramers-Brillouin (WKB) approximation. The agreement between the experimental data and the numerical model in Fig. $6 \mathrm{a}$ also highlights the importance of the atomistic modeling. The performance will continue to improve as the side gate is brought closer to the GNR, while preventing leakage. In addition, the performance will continue to improve if the width is scaled down from the $\sim 10 \mathrm{~nm}$ to $\sim 5 \mathrm{~nm}$, but it will start degrading if the GNR width is scaled down even lower. This is because the $I_{\text {off }}$ decreases when the GNR is scaled to smaller widths exponentially as $\sim 1 / \mathrm{W}$, but the interband tunneling oncurrent $I_{\text {on }}$ decreases with the GNR width exponentially.
To conclude, we have experimentally demonstrated roomtemperature operation of atomically thin GNR TFETs. Every feature in the operating characteristics can be understood from a combination of lithographically defined bandgaps and gated interband Easki and Zener tunneling current flow. The measured NDR tunneling current density is $\sim 1 \mathrm{~mA} / \mu \mathrm{m}$ shown in Fig. 4 . The larger-than-intended drain voltages necessary are significantly limited by contact and access resistances, as discussed in the Supplementary Materials. The on/off ratio is limited by both the contacts and the wide GNR width. The measured switching performance of these GNR TFETs at this stage are a far cry from what may be considered attractive for realistic applications; scaling of the GNR width and lower contact resistances are predicted to take the GNR-TFET performance close to those predicted in refs. 27,28 However, the observation of roomtemperature gate-tunable NDR in GNR channels is remarkable in its own right. In addition to proving the concept of atomically thin 1D TFETs based on GNRs, the shortcomings of the device reported here also clearly lay down the challenges ahead. Indeed, the control of narrow GNR widths on hBN with controlled edges and orientations, although challenging, was recently reported, ${ }^{46,47}$ which would be beneficial to exploit the inherent advantages of GNRs. The single most challenging task at this juncture to which no clear solution is in sight is to dope the GNR by taking advantage of the broken bonds at the edges. This step would mark a significant breakthrough, because it will not only enable completely controlled GNR TFETs, but also the simpler GNR-FETs -the ideal nanoscale transistors that carbon nanotubes have promised all along.

\section{METHODS}

Device fabrication

The GNRs were fabricated by electron-beam lithography (EBL). Hydrogen silsesquioxane (HSQ) diluted with methylisobutylketone is used as a negative electron-beam resist to pattern the GNRs and the details of the entire EBL process have been discussed in previous publications. ${ }^{33,48}$ Figure S1a shows the width of patterned HSQ lines as a function of the electron-beam dose. The width from the scanning electron microscope image in Fig. S1b is proportional to the line dose of the electron beam. After etching away the uncovered graphene by $\mathrm{O}_{2}$ plasma, the GNR width remains sub- $10 \mathrm{~nm}$, showing that the HSQ width is successfully transferred onto the graphene layer. $\mathrm{Cr}(5 \mathrm{~nm}) / \mathrm{Au}(150 \mathrm{~nm})$ were deposited by e-beam evaporation to form source/drain (S/D), side-gate, and back-gate metal electrodes. The S/D metals sit on top of the zero-bandgap 2D graphene regions to decrease the contact resistance. 


\section{Electrostatic doping of GNRs}

Unless otherwise stated, all materials were obtained from Sigma Aldrich. One gram of PEO $\left(M_{w} 100,000 \mathrm{~g} / \mathrm{mol}\right)$ was dissolved in $38 \mathrm{~g}$ of acetonitrile by mixing with a Teflon stir bar in a Teflon beaker. As commercial PEO contains micrometer-sized particles of $\mathrm{SiO}_{2}$, the solution is purified by centrifugation. To purify, the solution is transferred to two $50 \mathrm{~mL}$ centrifuge tubes (VWR) and centrifuged at $19,000 \times g$ for 5 min. The transparent supernatant is returned to the Teflon beaker, whereas the precipitate is discarded. Battery-grade $\mathrm{LiClO}_{4}(0.12 \mathrm{~g} ; 99.99 \%$ purity) is added to the $\mathrm{PEO} /$ acetonitrile solution and stirred for several hours. This PEO: $\mathrm{LiClO}_{4}$ ratio corresponds to an ether oxygen to $\mathrm{Li}$ ratio of 20:1. The solution is spin-coated onto the GNR-TFET at a spin-speed of 1000 RPM for $30 \mathrm{~s}$ and annealed for $5 \mathrm{~min}$ at $95^{\circ} \mathrm{C}$. This anneal temperature is well above the melting temperature $\left(60^{\circ} \mathrm{C}\right)$ of the polymer electrolyte at an ether oxygen to lithium ratio of $20: 1 .^{49}$ The sample is cooled to room temperature by removing it from the hotplate and placing it directly on a cleanroom wipe on the counter top. The thickness of the (PEO) ${ }_{20}: \mathrm{LiClO}_{4}$ film is $100 \mathrm{~nm}$, measured by ellipsometry. The side-gate metal and the electrolyte act as electrostatic doping agents by manipulating the electric potential of GNRs through mobile $\mathrm{Li}+$ and $\mathrm{ClO}_{4}^{-}$ions in PEO, thus creating a $p-n$ junction.

\section{Analytical model approach}

We have used a simple drift-diffusion analytical model to capture the correct electrostatics of the problem (Supplementary Fig. S4), before applying a full quantum transport + electrostatics to explain the expeimentally measured device characteristics. The current is the integral of the charge and mobility over the entire channel length. The charge density at a specific location is calculated based on the channel potential and density of states of graphene. The channel potential is solved based on the chargeneutrality equation.

\section{Atomic non-equilibrium Green's function approach}

The experimental data were also correlated with an atomistic nonequilibrium Green's function approach based on a p/d orbital tight-binding $\left(p / d\right.$ TB) model ${ }^{50}$ in which $\left\{p_{z \prime} d_{y z}, d_{z x}\right\}$ orbitals are included (Supplementary Fig. S5). Although there have been several theoretical studies about GNR TFETs, most of them are limited to the simple $p_{z}$ orbital tight-binding $\left(p_{z}\right.$ TB) model ${ }^{51,52}$ in which only the $p_{z}$ orbital is included in calculations and some of them are limited to the WKB approximations. ${ }^{28}$ The simple $p_{z}$ TB model only works for $2 \mathrm{D}$ graphene without considering edge effects. The $p / d$ TB model, however, is more accurate than the simple $p_{z}$ TB model in terms of the bandstructure, as it is calibrated to first-principle calculations and explicitly includes passivated hydrogen atoms in the Hamiltonian matrix. $^{50}$

\section{DATA AVAILABILITY}

All data derived from the experiments and calculations of this study are available from the corresponding author upon reasonable request.

Received: 6 September 2017; Accepted: 16 October 2019; Published online: 07 November 2019

\section{REFERENCES}

1. Novoselov, K. S. et al. Electric field effect in atomically thin carbon films. Science 306, 666-669, https://doi.org/10.1126/science.1102896 (2004).

2. Geim, A. K. \& Novoselov, K. S. The rise of graphene. Nat. Mater. 6, 183-191, https://doi.org/10.1142/9789814287005_0002 (2007).

3. Zhang, Y., Tan, Y.-W., Stormer, H. L. \& Kim, P. Experimental observation of the quantum Hall effect and Berry's phase in graphene. Nature 438, 201-204, https:// doi.org/10.1038/nature04235 (2005).

4. Jena, D. Tunneling transistors based on graphene and 2-D crystals. Proc. IEEE 101, 1585-1602, https://doi.org/10.1109/JPROC.2013.2253435 (2013).

5. Zhao, P., Feenstra, R. M., Gu, G. \& Jena, D. SymFET: a proposed symmetric graphene tunneling field-effect transistor. IEEE T. Electron. Dev. 60, 951-957, https:// doi.org/10.1109/TED.2013.2238238 (2013).

6. Mishchenko, A. et al. Twist-controlled resonant tunneling in graphene/boron nitride/graphene heterostructures. Nat. Nanotechnol. 9, 808-813, https://doi.org/ 10.1038/nnano.2014.187 (2014).
7. Nagashima, A., Tejima, N., Gamou, Y., Kawai, T. \& Oshirna, C. Electronic structure of monolayer hexagonal boron nitride physisorbed on metal surfaces. Phys. Rev. Lett. 75, 3918-3921, https://doi.org/10.1103/PhysRevLett.75.3918 (1995).

8. Watanabe, K., Taniguchi, T. \& Kanda, H. Direct-bandgap properties and evidence for ultraviolet lasing of hexagonal boron nitride single crystal. Nat. Mater. 3, 404-409, https://doi.org/10.1038/nmat1134 (2004).

9. Dean, C. R. et al. Boron nitride substrate for high-quality graphene electronics. Nat. Nanotechnol. 5, 722-726, https://doi.org/10.1038/nnano.2010.172 (2010).

10. Meric, l. et al. Graphene field-effect transistors based on boron-nitride dielectrics. Proc. IEEE 101, 1609-1619, https://doi.org/10.1109/JPROC.2013.2257634 (2013).

11. Jang, S. K., Youn, J., Song, Y. J. \& Lee, S. Synthesis and characterization of hexagonal boron nitride as a gate dielectric. Sci. Rep. 6, 30449, https://doi.org/ 10.1038/srep30449 (2016).

12. Radisavljevic, B., Radenovic, A., Brivio, J., Giacometti, V. \& Kis, A. Single-layer $\mathrm{MoS}_{2}$ transistors. Nat. Nanotechnol. 6, 147-150, https://doi.org/10.1021/nn2024557 (2011).

13. Kim, S. et al. High-mobility and low-power thin-film transistors based on multilayer $\mathrm{MoS}_{2}$ crystals. Nat. Commun. 3, 1011, https://doi.org/10.1038/ncomms2018 (2012).

14. Li, X. et al. Controlled vapor phase growth of single crystalline, two-dimensional GaSe crystals with high photoresponse. Sci. Rep. 4, 5497, https://doi.org/10.1038/ srep05497 (2014).

15. Ohta, T., Bostwick, A., Seyller, T., Horn, K. \& Rotenberg, E. Controlling the electronic structure of bilayer graphene. Science 313, 951-954, https://doi.org/ 10.1126/science.1130681 (2006).

16. Zhang, Y. et al. Direct observation of a widely tunable bandgap in bilayer graphene. Nature 459, 820-823, https://doi.org/10.1038/nature08105 (2009).

17. Rudenko, A. N. \& Katsnelson, M. I. Quasiparticle band structure and tight-binding model for single- and bilayer black phosphorus. Phys. Rev. B 89, 201408(R), https://doi.org/10.1103/PhysRevB.89.201408 (2014).

18. Lu, J. et al. Bandgap engineering of phosphorene by laser oxidation toward functional 2D materials. ACS Nano 9, 10411-10421, https://doi.org/10.1021/ acsnano.5b04623 (2015).

19. Charlier, J.-C., Blas, X. \& Roche, S. Electronic and transport properties of nanotubes. Rev. Mod. Phys. 79, 677-732, https://doi.org/10.1103/RevModPhys.79.677 (2007).

20. Franklin, A. D. et al. Sub-10 nm carbon nanotube transistor. Nano Lett. 12, 758-762, https://doi.org/10.1021/nl203701g (2012).

21. Arnold, M. S., Green, A. A., Hulvat, J. F., Stupp, S. I. \& Hersam, M. C. Sorting carbon nanotubes by electronic structure using density differentiation. Nature 1, 60-65, https://doi.org/10.1038/nnano.2006.52 (2006).

22. Wang, C. et al. Wafer-scale fabrication of separated carbon nanotube thin-film transistors for display applications. Nano Lett. 9, 4285-4291, https://doi.org/ 10.1021/nl902522f (2009).

23. Appenzeller, J., Lin, Y.-M., Knoch, J. \& Avouris, Ph Band-to-band tunneling in carbon nanotube field-effect transistors. Phys. Rev. Lett. 93, 196805, https://doi. org/10.1103/PhysRevLett.93.196805 (2004).

24. Ionescu, A. M. \& Riel, H. Tunnel field-effect transistors as energy efficient electronic switches. Nature 479, 329-337, https://doi.org/10.1038/nature10679 (2011).

25. Ma, N. \& Jena, D. Interband tunneling in two-dimensional crystal semiconductors. Appl. Phys. Lett. 102, 132102, https://doi.org/10.1063/1.4799498 (2013).

26. Jena, D., Fang, T., Zhang, Q. \& Xing, H. Zener tunneling in semiconducting nanotube and graphene nanoribbon $p-n$ junctions. Appl. Phys. Lett. 93, 112106, https://doi.org/10.1063/1.2983744 (2008).

27. Nikonov, D. E. \& Young, I. A. Benchmarking of beyond-CMOS exploratory devices for logic integrated circuits. IEEE J. Explor. Solid State Computat. Devices Circuits 1, 1-11, https://doi.org/10.1109/JXCDC.2015.2418033 (2015).

28. Zhang, Q., Fang, T., Xing, H., Seabaugh, A. \& Jena, D. Graphene nanoribbon tunnel transistors. IEEE Electr. Device Lett. 29, 1344-1346, https://doi.org/10.1109/ LED.2008.2005650 (2008).

29. Han, M. Y., Özyilmaz, B., Zhang, Y. \& Kim, P. Energy band-gap engineering of graphene nanoribbons. Phys. Rev. Lett. 98, 206805, https://doi.org/10.1103/ PhysRevLett.98.206805 (2007).

30. Li, X. et al. Large-area synthesis of high-quality and uniform graphene films on copper foils. Science 324, 1312, https://doi.org/10.1126/science.1171245 (2009).

31. Liao, A. D. et al. Thermally limited current carrying ability of graphene nanoribbons. Phys. Rev. Lett. 106, 256801, https://doi.org/10.1103/PhysRevLett.106.256801 (2011).

32. Hwang, W. S. et al. Graphene nanoribbon field-effect transistors on wafer-scale epitaxial graphene on SiC substrates. APL Mater. 3, 011101, https://doi.org/ 10.1063/1.4905155 (2015).

33. Hwang, W. S. et al. Transport properties of graphene nanoribbon transistors on chemical-vapor-deposition grown wafer-scale graphene. Appl. Phys. Lett. 100, 203107, https://doi.org/10.1063/1.4716983 (2012). 
34. Nilsson, H. A. et al. Temperature dependent properties of $\ln \mathrm{Sb}$ and $\ln \mathrm{As}$ nanowire field-effect transistors. Appl. Phys. Lett. 96, 153505, https://doi.org/10.1063/ 1.3402760 (2010).

35. Lee, H. et al. Sub-5 nm all-around gate FinFET for ultimate scaling. 2006 Symposium on VLSI Technology. Digest of Technical Papers. 58-59, https://doi.org/ 10.1109/VLSIT.2006.1705215 (2006).

36. Williams, J. R., DiCarlo, L. \& Marcus, C. M. Quantum Hall effect in a gate-controlled $p-n$ junction of graphene. Science 317, 638, https://doi.org/10.1126/ science.1144657 (2007)

37. Farmer, D. B., Lin, Y. -M., Afzali-Ardakani, A. \& Avouris, P. Behavior of a chemically doped graphene junction. Appl. Phys. Lett. 94, 213106, https://doi.org/10.1063/ 1.3142865 (2009).

38. Huard, B., Stander, N., Sulpizio, J. A. \& Goldhaber-Gordon, D. Evidence of the role of contacts on the observed electron-hole asymmetry in graphene. Phys. Rev. $B$ 78, 121401(R), https://doi.org/10.1103/PhysRevB.78.121402 (2008)

39. Romero, H. E. et al. N-type behavior of graphene supported on $\mathrm{Si} / \mathrm{SiO}_{2}$ substrates. ACS Nano 2, 2037-2044, https://doi.org/10.1021/nn800354m (2008).

40. Wang, $X$. et al. N-doping of graphene through electrothermal reactions with ammonia. Science 324, 768-771, https://doi.org/10.1126/science.1170335 (2009).

41. Ueno, K. et al. Electric-field-induced superconductivity in an insulator. Nat. Mater. 7, 855, https://doi.org/10.1038/nmat2298 (2008)

42. Welton, T. Room-temperature ionic liquids. Solvents for synthesis and catalysis. Chem. Rev. 99, 2071, https://doi.org/10.1021/cr980032t (1999).

43. Ye, Y.-S. et al. lonic liquid polymer electrolytes. J. Mater. Chem. A 1, 2719, https:// doi.org/10.1039/C2TA00126H (2013).

44. Efetov, D. K. \& Kim, P. Controlling electron-phonon interactions in graphene at ultrahigh carrier densities. Phys. Rev. Lett. 105, 256805, https://doi.org/10.1103/ PhysRevLett.105.256805 (2010).

45. Luisier, M. \& Klimeck, G. Performance analysis of statistical samples of graphene nanoribbon tunneling transistors with line edge roughness. Appl. Phys. Lett. 94 223505, https://doi.org/10.1063/1.3140505 (2009).

46. Chen, L. et al. Oriented graphene nanoribbons embedded in hexagonal boron nitride trenches. Nat. Commun. 8, 14703, https://doi.org/10.1038/ncomms14703 (2017).

47. Che, L. et al. Edge control of graphene domains grown on hexagonal boron nitride. Nanoscale 9, 11475, https://doi.org/10.1039/C7NR02578E (2017).

48. Hwang, W. S. et al. Fabrication of top-gated epitaxial graphene nanoribbon FETs using hydrogen-silsesquioxane. J. Val. Sci. Technol. B 30, 03D104, https://doi.org/ 10.1116/1.3693593 (2012)

49. Robitaille, C. \& Fauteus, D. Phase-diagrams and conductivity characterization of some PEO-Lix electrolytes. J. Elec. Soc. 133, 315, https://doi.org/10.1149/ 1.2108569 (1986)

50. Boykin, T. B. et al. Accurate six-band nearest-neighbor tight-binding model for the $\pi$-bands of bulk graphene and graphene nanoribbons. J. Appl. Phys. 109, 104304, https://doi.org/10.1063/1.3582136 (2011).

51. Luisier, M. \& Klimeck, G. Performance analysis of statistical samples of graphene nanoribbon tunneling transistors with line edge roughness. Appl. Phys. Lett. 94, 223505, https://doi.org/10.1063/1.3140505 (2011).

52. Fiori, G. \& lannaccone, G. Simulation of graphene nanoribbon field-effect transistors. IEEE Elec. Dev. Lett. 28, 760, https://doi.org/10.1109/LED.2007.901680 (2007).

\section{ACKNOWLEDGEMENTS}

This research made use of the Cornell Nanofabrication Facility and the Center for Nano Science and Technology at University of Notre Dame, which were supported in part by the Semiconductor Research Corporation (SRC), Nanoelectronics Research
Initiative (NRI), and the National Institute of Standards and Technology (NIST) through the Midwest Institute for Nanoelectronics Discovery (MIND), STARnet, an SRC program sponsored by MARCO and DARPA, and by the Office of Naval Research (ONR) and the National Science Foundation (NSF). The authors acknowledge the single layer graphene provided by Dr Chun-Yung Sung (from the IBM T. J. Watson Center at the time). The authors also acknowledge the nanoHUB.org computing resources provided by the National Institute for Computational Sciences (NICS) and by Network for Computational Nanotechnology funded by the National Science Foundation. W.S.H. is grateful to Center for Advanced Soft Electronics funded by the Ministry of Science, ICT, and Future Planning as Global Frontier Project (CASE-20110031638) and Basic Science Research Program (2017R1A2B4012278) by the National Research Foundation of Korea (NRF).

\section{AUTHOR CONTRIBUTIONS}

W.S.H., A.S. and D.J. designed the experiment. W.S.H., R.Y. and S.K.F.-S. fabricated and prepared the samples. P.Z., S.G.K., G.K. and D.J. performed the models and simulations. W.S.H., G.K., A.S., S.K.F.-S., H(G).X. and D.J. analysed the data. W.S.H., S. G.K, G.K., S.K.F.-S. and D.J. wrote the manuscript. All authors discussed the data and contributed to the manuscript.

\section{COMPETING INTERESTS}

The authors declare no competing interests.

\section{ADDITIONAL INFORMATION}

Supplementary information is available for this paper at https://doi.org/10.1038/ s41699-019-0127-1.

Correspondence and requests for materials should be addressed to W.S.H. or D.J.

Reprints and permission information is available at http://www.nature.com/ reprints

Publisher's note Springer Nature remains neutral with regard to jurisdictional claims in published maps and institutional affiliations.

\begin{abstract}
Open Access This article is licensed under a Creative Commons Attribution 4.0 International License, which permits use, sharing, adaptation, distribution and reproduction in any medium or format, as long as you give appropriate credit to the original author(s) and the source, provide a link to the Creative Commons license, and indicate if changes were made. The images or other third party material in this article are included in the article's Creative Commons license, unless indicated otherwise in a credit line to the material. If material is not included in the article's Creative Commons license and your intended use is not permitted by statutory regulation or exceeds the permitted use, you will need to obtain permission directly from the copyright holder. To view a copy of this license, visit http://creativecommons org/licenses/by/4.0/.
\end{abstract}

C The Author(s) 2019 